$\mathrm{BNL}-694.14$

Informal Report

\title{
The Algebra of Taylor Series and the Roots of a General Polynomial
}

\author{
John Herrera
}

October 11, 2002

Superconducting Magnet Division

\author{
Brookhaven National Laboratory \\ Operated by \\ Brookhaven Science Associates \\ Upton, NY 11973 \\ Under Contract with the United States Department of Energy \\ Contract Number DE-AC02-98CH10886
}




\section{DISCLAIMER}

This report was prepared as an account of work sponsored by an agency of the United States Government. Neither the United States Government nor any agency thereof, nor any of their employees, nor any of their contractors, subcontractors or their employees, makes any warranty, express or implied, or assumes any legal liability or responsibility for the accuracy, completeness, or any third party's use or the results of such use of any information, apparatus, product, or process disclosed, or represents that its use would not infringe privately owned rights. Reference herein to any specific commercial product, process, or service by trade name, trademark, manufacturer, or otherwise, does not necessary constitute or imply its endorsement, recommendation, or favoring by the United States Government or any agency thereof or its contractors or subcontractors. The views and opinions of authors expresses herein do not necessarily state to reflect those of the United States Government or any agency thereof. 
BNL-69414

Informal Report

The Algebra of Taylor Series and the Roots of a General Polynomial

John Herrera

October 11, 2002 


\title{
The Algebra of Taylor Series and the Roots of a General Polynomial
}

\author{
J. C. Herrera \\ Brookhaven National Laboratory, Upton, NY 11973-5000 (Retired)
}

\begin{abstract}
We develop the basic elements of the algebra of Taylor series. This knowledge allows us to derive a series expression for an exact root of a general polynomial of arbitrary degree.
\end{abstract}

\section{Introduction:}

Taylor series are commonly used in the mathematical analysis of physical systems. It is our purpose in this paper to present systematically the algebra of Taylor series, and then to use this knowledge to derive an expression for an exact root of a polynomial of arbitrary degree.

Drawing on the discussion of Taylor series as it appears in Whittaker and Watson [1], we write the expansion of a function about an offset $z_{0}$ as

$$
f(z)=\sum_{n=0}^{\infty} a_{n}\left(z_{0}\right)\left(z-z_{0}\right)^{n}
$$

All the quantities in Eq. (1) are complex and the coefficients are defined by the contour integral centered at $z_{0}$

$$
a_{n}\left(z_{0}\right)=\frac{1}{2 \pi i} \oint \frac{f(z) d z}{\left(z-z_{0}\right)^{n+1}}, \quad n=0,1,2, \ldots
$$

The radius of convergence for the Taylor series is the lower bound

$$
R_{a}=\varliminf^{\lim }\left|a_{n}\left(z_{0}\right)\right|^{-1 / n}, \quad n \rightarrow \infty \text {. }
$$

\section{Algebraic structure for addition and multiplication:}

Our approach to the algebraic properties of the set of Taylor series of the form of Eq. (1) is entirely based on Eq. (2) for the coefficients of each element of the set. Thus with respect to the binary operation of addition, it is clear that such a set constitutes an additive abelian group. The identity element for additon is the element with coefficients $a_{n}\left(z_{0}\right)=0$. Similarly, as we shall verify in the following two sections, the set of elements characterized by having $a_{0}\left(z_{0}\right) \neq 0$, form with respect to multiplication, a multiplicative abelian group with the identity: $a_{n}\left(z_{0}\right)=(1,0,0, \ldots)$. Since the distributive law over addition also holds, we can say that the set of Taylor series expanded about a given point $z_{0}$ constitutes an infinite field over the field of complex numbers $[2,3]$. 


\section{Product of two Taylor series:}

As a simple illustration of the method of basing the analysis on Eq. (2), we consider the product of the series $f(z)$ with another one $g(z)$. Thus,

$$
h(z)=f(z) g(z)=\sum_{k=0}^{\infty} a_{k}\left(z-z_{0}\right)^{k} \sum_{\ell=0}^{\infty} b_{\ell}\left(z-z_{0}\right)^{\ell},
$$

or

$$
h(z)=\sum_{n=0}^{\infty} c_{n}\left(z-z_{0}\right)^{n}=\sum_{k=0}^{\infty} \sum_{\ell=0}^{\infty} a_{k} b_{\ell}\left(z-z_{0}\right)^{k+\ell} .
$$

Dividing Eq. (5) by the quantity $\left(z-z_{0}\right)$ and performing the contour integration yields

$$
\frac{1}{2 \pi i} \oint \frac{f(z) g(z) d z}{z-z_{0}}=\sum_{k=0}^{\infty} \sum_{\ell=0}^{\infty} a_{k} b_{\ell} \delta_{k+\ell, 0} .
$$

Here $\delta_{k+\ell, 0}$ is the Kronecker delta function equal to one when $(k+\ell)=0$ and zero otherwise. Consequently, the zero degree coefficient in the expansion of $h(z)$ is, as expected,

$$
c_{0}=a_{0} b_{0} .
$$

Moving on to the $n^{\text {th }}$ degree coefficient, we derive in a similar way

$$
\frac{1}{2 \pi i} \oint \frac{f(z) g(z) d z}{\left(z-z_{0}\right)^{n+1}}=\sum_{k=0}^{\infty} \sum_{\ell=0}^{\infty} a_{k} b_{\ell} \delta_{k+\ell, n}=\sum_{k=0}^{n} a_{k} b_{n-k},
$$

Or, more explicitly,

$$
c_{n}\left(z_{0}\right)=\sum_{k=0}^{n} a_{k}\left(z_{0}\right) b_{n-k}\left(z_{0}\right), \quad n=0,1,2, \ldots
$$

It is important to point out that in the derivation of the recursion relation for the coefficients, Eq. (9), we have dealt with three series that are all expanded about the same non-singular offset point $z_{0}$. The product expansion converges within its own radius of convergence, $R_{c}$, specified by Eq. (3) and centered at $z_{0}$.

\section{Reciprocal of a Taylor series:}

We next consider the reciprocal of $f(z)$

$$
f^{-1}(z)=\sum_{\ell=0}^{\infty} b_{\ell}\left(z-z_{0}\right)^{\ell} .
$$

Since the identity

$$
\frac{1}{2 \pi i} \oint \frac{f(z) f^{-1}(z) d z}{\left(z-z_{0}\right)^{n}}=\frac{1}{2 \pi i} \oint \frac{d z}{\left(z-z_{0}\right)^{n}}=\delta_{n, 1}, n \geq 1,
$$


is valid, it follows that

$$
\frac{1}{2 \pi i} \oint \frac{d z f(z)}{\left(z-z_{0}\right)^{n}} \sum_{\ell=0}^{\infty} b_{\ell}\left(z-z_{0}\right)^{\ell}=\delta_{n, 1} .
$$

Therefore,

$$
\sum_{\ell=0}^{n-1} b_{\ell} a_{n-\ell-1}=\delta_{n, 1} .
$$

This recursion relation specifies that the successive values of the coefficients for the reciprocal of Eq. (1) are

and

$$
\begin{gathered}
b_{0}=1 / a_{0}, \quad a_{0} \neq 0, \\
b_{n}=-\frac{1}{a_{0}} \sum_{\ell=1}^{n} b_{n-\ell} a_{\ell}, \quad n \geq 1 .
\end{gathered}
$$

\section{A Taylor series taken to a power:}

Let us examine the non-binary operation of exponentiation,

or

$$
\begin{gathered}
g(z)=[f(z)]^{\alpha}, \\
\sum_{k=0}^{\infty} b_{k}\left(z-z_{0}\right)^{k}=\left[\sum_{n=0}^{\infty} a_{n}\left(z-z_{0}\right)^{n}\right]^{\alpha}, \quad a_{0} \neq 0 .
\end{gathered}
$$

The zero degree coefficient for $g(z)$ is evidently

$$
b_{0}=g\left(z_{0}\right)=\left[a_{0}\right]^{\alpha} .
$$

Differentiating Eq. (15) with respect to $z$ gives

$$
f(z) g^{\prime}(z)=\alpha g(z) f^{\prime}(z),
$$

and, as a result,

$$
f(z) \sum_{k=1}^{\infty} k b_{k}\left(z-z_{0}\right)^{k-1}=\alpha g(z) \sum_{k=1}^{\infty} k a_{k}\left(z-z_{0}\right)^{k-1} .
$$

A division by the quantity $\left(z-z_{0}\right)^{\ell}$ followed by a contour integration leads to the equality

$$
\sum_{k=1}^{\infty} k b_{k} \oint \frac{f(z) d z}{\left(z-z_{0}\right)^{\ell-k+1}}=\alpha \sum_{k=1}^{\infty} k a_{k} \oint \frac{g(z) d z}{\left(z-z_{0}\right)^{\ell-k+1}} .
$$

Whence, the recursion relationship assumes the form

$$
\sum_{k=1}^{\ell} k b_{k} a_{\ell-k}=\alpha \sum_{k=1}^{\ell} k a_{k} b_{\ell-k},
$$


with the result that

$$
b_{1} a_{0}=\alpha a_{1} b_{0}, \quad \ell=1,
$$

and, for $\ell \geq 2$

$$
b_{\ell} a_{0}=\alpha a_{\ell} b_{0}+\sum_{k=1}^{\ell-1} \frac{(\ell-k) \alpha-k}{\ell} a_{\ell-k} b_{k} .
$$

We observe that since the exponent $\alpha$ has not been restricted to integers, the coefficient $b_{0}$, Eq. (17), may be multivalued and corresponds to different elements of the set of Taylor series, each equally valid.

\section{Reversion of a Taylor series:}

The series in Eq. (1) converges over a region in the complex $z$ plane centered at $z=z_{0}$. Instead, the reversion of $f(z)$ will be a series in the complex $f$ plane centered at the offset $a_{0}=f\left(z_{0}\right)$. Accordingly, the reversion series is expressed as

$$
z(f)=\sum_{n=0}^{\infty} b_{n}\left(a_{0}\right)\left(f-a_{0}\right)^{n},
$$

with

$$
z\left(a_{0}\right)=b_{0}\left(a_{0}\right)=z_{0} .
$$

We note that this series is expanded about the point $a_{0}$, and therefore it does not belong to our original set expanded about $z_{0}$. Eq. (23) is rewritten in the convenient expanded form

$$
z(f)=z_{0}+\sum_{n=1}^{\infty} b_{n}\left(a_{0}\right)\left(f-a_{0}\right)^{n}
$$

Our task at this point is that of expressing the $b_{n}\left(a_{0}\right)$ coefficients in terms of the $a_{n}\left(z_{0}\right)$ coefficients of Eq. (1). To accomplish this end, we write

$$
f-a_{0}=a_{1}\left(z-z_{0}\right) g(z),
$$

where the function

$$
g(z)=\sum_{k=0}^{\infty} A_{k}\left(z-z_{0}\right)^{k},
$$

has the coefficients

$$
A_{0}=1 ; \quad A_{k}=a_{k+1} / a_{1}, \quad k \geq 1 .
$$

Since the differential relation

$$
z^{\prime}(f) d f=d z
$$

holds, it is evident that 


$$
\frac{z^{\prime}(f) d f}{\left(f-a_{0}\right)^{\ell}}=\frac{d z}{\left(f-a_{0}\right)^{\ell}} .
$$

Integration of both sides of Eq. (30) about the corresponding points $a_{0}$ and $z_{0}$ results directly in

$$
\oint \frac{z^{\prime}(f) d f}{\left(f-a_{0}\right)^{\ell}}=\oint \frac{d z}{a_{1}^{\ell}\left(z-z_{0}\right)^{\ell}}[g(z)]^{\ell} .
$$

When we develop that last factor in Eq. (31) into a Taylor series

$$
[g(z)]^{-\ell}=\sum_{k=0}^{\infty} B(\ell, k)\left(z-z_{0}\right)^{k},
$$

and realize that the left-hand side of Eq. (31) is the Taylor coefficient for the derivative of $z(f)$, we arrive at the equation

$$
\ell b_{\ell}=B(\ell, \ell-1) / a_{1}^{\ell} .
$$

Because Eq. (32) is itself the $(-\ell)$-th power of a Taylor series, we can explicitly, per Eq. (22), write $B(\ell, k)$ as the sequence:

$$
\begin{gathered}
B(\ell, 0)=1, \\
B(\ell, 1)=-\ell\left(\frac{a_{2}}{a_{1}}\right), \\
\vdots \\
B(\ell, k)=-\ell\left(\frac{a_{k+1}}{a_{1}}\right)-\sum_{j=1}^{k-1} \frac{(k-j) \ell+j}{k}\left(\frac{a_{k-j+1}}{a_{1}}\right) B(\ell, j), \\
\vdots \\
B(\ell, \ell-1)=-\ell\left(\frac{a_{\ell}}{a_{1}}\right)-\sum_{j=1}^{\ell-2}(\ell-j)\left(\frac{a_{\ell-j}}{a_{1}}\right) B(\ell, j) .
\end{gathered}
$$

In summary, the reversion series is Eq. (25) with the $b_{n}\left(a_{0}\right)$ coefficients dependent upon the original [see Eq. (1)] $a_{n}\left(z_{0}\right)$ coefficients through Eq. (34d). Briefly then

$$
z(f)=z_{0}+\sum_{\ell=1}^{\infty} \frac{B(\ell, \ell-1)}{\ell\left[a_{1}\left(z_{0}\right)\right]^{\ell}}\left[f-a_{0}\left(z_{0}\right)\right]^{\ell} .
$$

We have not found in the literature the recursion relations for the reversion of a Taylor series. This observation is consistent with the statements made by K. Knopp in his two texts $[4,5]$ that the recursion relations are unknown. 


\section{Root of a polynomial of arbitrary degree:}

A polynomial is a Taylor series characterized by $a_{n}\left(z_{0}\right)=0$ above some term of degree $N$, that is

$$
f(z)=\sum_{n=0}^{N} a_{n}\left(z_{0}\right)\left(z-z_{0}\right)^{n}, \quad a_{0}\left(z_{0}\right) \neq 0 .
$$

In agreement with the previous development, such a series has a corresponding reversion series of the form of Eq. (35). Under the condition that the value of the polynomial vanishes $f=0$, Eq. (35) becomes an explicit expression for the root, $z(0)$,

$$
z(0)=z_{0}+\sum_{\ell=1}^{\infty} \frac{B(\ell, \ell-1)}{\ell}\left[-\frac{a_{0}\left(z_{0}\right)}{a_{1}\left(z_{0}\right)}\right]^{\ell}
$$

It is important to mention that the right-hand side of this formula depends only on the value of the offset $z_{0}$. It will be the root $z(0)$ when the value, $f=0$, is within the circle of convergence of the reversion series, Eq. (35), centered at $a_{0}\left(z_{0}\right)$ in the complex $f$ plane.

It behooves us, in view of this situation, to locate an appropriate offset $z_{0}$ in the complex $z$ plane. We therefore give, in the following, one way of doing this. To start, let us assume that we are initially given a polynomial having $N$ distinct roots distributed arbitrarily in the complex $z$ plane about the origin, $z_{0}=0$, of the coordinate system. Therefore

$$
f(z)=\sum_{n=0}^{N} a_{n}(0) z^{n}, a_{0}(0) \neq 0 .
$$

We now carry out the following three numerical manipulations:

1. With the aid of Eqs. $(14 a, b)$, we calculate the coefficients of the reciprocal of the given polynomial, Eq. (38).

2. Applying Eq. (3) up to some high-degree coefficient, we derive an estimate of the radius of convergence, $R_{b}$, of this reciprocal series.

3. We now numerically sweep at small angular intervals along this approximate circle of convergence searching for a complex value of $z$ for which the initial polynomial, Eq. (38), takes on its smallest absolute value. This value of $z$ is the complex offset $z_{0}$ that we seek.

At this juncture in our discussion we must emphasize that the successful outcome of our 3-step algorithm is that it give a value of $z_{0}$ such that the circle of convergence of Eq. (35) contains the value $f=0$. Subject to this condition, the particular complex value of $z_{0}$ is not critical. When the initial polynomial, Eq. (38), is displaced by this value of the offset $z_{0}$, it becomes

$$
f(z)=\sum_{n=0}^{N} a_{n}\left(z_{0}\right)\left(z-z_{0}\right)^{n}
$$


The coefficients $a_{n}\left(z_{0}\right)$ that appear in this displaced form of the polynomial are the ones we insert in Eq. (37), and thereby calculate $z(0)$, a root of Eq. (38).

\section{Summary and Conclusions:}

We have presented the basic elements of the algebra of Taylor series and verified that for the binary operations of addition and multiplication the set of Taylor series expanded about a point constitutes a field over the field of complex numbers. For the non-binary operations of exponentiation and reversion we have derived the appropriate recursion relations for the expansion coefficients. Section 7 has dealt with the problem of solving for a root of a general polynomial. The remaining roots are found by following, for each of them, the same procedure of locating the pertinent offset with the help of the 3-step algorithm, and then calculating the nearby root employing Eq. (37). As a check on the validity and generality of these ideas, we have written a computer program and successfully solved for the numerical roots of a large variety of polynomials of many degrees (up to $N=17$ ). The accuracy of these calculated roots has been equal to the expected machine precision of about one part in $10^{15}$. In these exercises, polynomials with roots of multiplicity greater than one have been reduced to equivalent ones that have the same roots but with a multiplicity of one. The procedure for carrying out this operation is clearly explained in reference [6].

In conclusion, we can state that Eq. (37), in conjunction with a method of locating an appropriate offset $z_{0}$, is an answer to the long-standing problem of finding an expression for the exact roots of a general polynomial of arbitrary degree. This statement does not contradict the "Impossibility Theorem" of Abel [7], since his landmark theorem addresses itself only to the specific question of which polynomials are solvable algebraically by radicals $[2,8,9]$. As a final remark, we would like to add that by "an exact root" we mean one that is obtainable directly from a formula, like Eq. (37), without employing successive approximations or iterations.

\section{Acknowledgements:}

I would like to thank the members of the Magnet Division, Brookhaven National Laboratory, for their continuing support. In particular, the many informative discussions that I have had with Animesh Jain and Erich Willen are sincerely appreciated.

\section{References:}

[1] E. T. Whittaker and G. N. Watson, A Course of Modern Analysis, (Cambridge University Press, American Edition, 1948.)

[2] John E. Maxfield and Margaret W. Maxfield, Abstract Algebra and Solution by Radicals, (Dover Publications, Inc., N.Y., 1971.)

[3] P. Henrici, Applied and Computational Analysis, Vol. 1, (John Wiley and Sons, Inc. 1974.)

[4] K. Knopp, Infinite Sequences and Series, p. 122, footnote 2, (Dover Publications, N.Y., 1956.) 
[5] K. Knopp, Theory and Application of Infinite Series, p. 187, (Dover Publications, N.Y., 1990.)

[6] A. P. Mishina and I. V. Proskuryakov, Higher Algebra, p. 162. (Pergamon Press, First English Edition, 1965.)

[7] N. H. Abel, Oeuvres Complètes, 1881 Tome Second, Memoire VII, pp. 66-94. (Johnson Reprint Corp, N.Y., 1995).

[8] A. Cayley, 'Equation', in Encyclopedia Britannica, 11 Edition, Vol. 9, p. 709 (1911)

[9] R. G. Ayoub, American Mathematical Monthly 89, 397 (1982). 